\title{
Does Primate Motion Perception Depend on the Magnocellular Pathway?
}

\author{
William H. Merigan, ${ }^{1,4}$ Carey E. Byrne, ${ }^{2, a}$ and John H. R. Maunse $\|^{3,4}$ \\ Departments of ${ }^{1}$ Ophthalmology, ${ }^{2}$ Neurobiology and Anatomy, and ${ }^{3 P h y s i o l o g y}$ and ${ }^{4}$ Center for Visual Science, University \\ of Rochester Medical Center, Rochester, New York 14642
}

This study examined the importance of the primate magnocellular retinocortical pathway in the perception of moving stimuli. A portion of the magnocellular pathway was permanently and selectively interrupted by ibotenic acid injections in the LGN of macaque monkeys. We then tested contrast sensitivity for detecting moving stimuli, as well as two indices of motion perception, contrast sensitivity for opposite direction discrimination and speed difference thresholds, in the affected portion of the visual field. Magnocellular lesions greatly reduced detection contrast sensitivity at high temporal and low spatial frequencies and had a similar effect on contrast sensitivity for opposite direction discrimination under these same stimulus conditions. Consequently, opposite direction discriminations could be made at contrast threshold, suggesting that magnocellular lesions reduced the visibility of stimuli used to test direction perception, but did not act directly on direction perception. Magnocellular lesions also elevated speed difference thresholds under some stimulus conditions. However, this deficit was reduced or eliminated by raising the contrast of the test stimulus. Together, these findings suggest that magnocellular lesions reduce the visibility of stimuli used to test motion perception but that they do not appear to alter motion perception otherwise.

The visual pathway through magnocellular layers of the lateral geniculate is thought to play an important role in motion processing for several reasons. This pathway dominates visual sensitivity at low spatial and high temporal frequencies (Merigan and Eskin, 1986; Merigan and Maunsell, 1990), conditions that are most favorable for motion perception (Watson et al., 1980; Pasternak, 1987). Cortical projections of the magnocellular pathway (M pathway) provide the major input to cortical areas MT and MST (Maunsell and Newsome, 1987; DeYoe and Van Essen, 1988), which appear to be important for motion processing (Movshon et al., 1985; Newsome and Paré, 1988; Merigan et al., 1991b). In a recent study (Maunsell et al., 1990), it was shown that the response of neurons in cortical area MT is

\footnotetext{
Received Mar. 11, 1991; revised May 31, 1991; accepted June 6, 1991

Wc thank Cheryl Ruff-Neri and James Jester for assistance in testing monkeys and Peter Vamvakius for histology and lesion reconstruction. Drs. Tatiana Pasternak and Richard Aslin commented on the manuscript. This research was supported by Grants ES01247, EY01319, EY05911, and AFOSR 89-0041.

Correspondence should be addressed to William H. Merigan, Box 314, University of Rochester Medical Center, Rochester, NY 14642.

a Present address: Gerontology Research Center, NIA/NIH, 4940 Eastern Avenue, Baltimore, MD 21224.

Copyright (c) 1991 Society for Neuroscience $0270-6474 / 91 / 113422-08 \$ 05.00 / 0$
}

dominated by input from the $\mathbf{M}$ pathway. Finally, it was demonstrated (Schiller et al., 1990) that lesions of the M pathway in monkeys eliminated the detection of motion of a group of dots in a field of random dots.

On the other hand, a careful reading of even the above articles suggests caution in attributing motion perception exclusively to the M pathway. DeYoe and Van Essen (1988) summarize the evidence of mixing of parvocellular and magnocellular streams within visual cortex. Moreover, Maunsell et al. (1990) have demonstrated moderate parvocellular input to cortical area MT, which may be important for motion processing.

The present study used ibotenic acid lesions of magnocellular layers of the lateral geniculate to study the role of this pathway in detection contrast sensitivity for moving stimuli and in the performance of two psychophysical tasks that are thought to reflect motion processing. The first is the contrast sensitivity for discriminating opposite directions of motion of drifting gratings, and the second is speed difference thresholds obtained with drifting gratings. The results of these studies suggest a new view of the role of the $\mathrm{M}$ pathway in motion perception, namely, that it mediates the high visibility of the rapidly moving stimuli that are often used to test motion perception, but that motion perception is not dependent on this pathway.

\section{Materials and Methods}

\section{Subjects}

The subjects were three adult, female monkeys (Macaca nemestrina) of approximately $5 \mathrm{~kg}$ body weight. They had free access to monkey chow, supplemented regularly with fresh fruit, and their water was withheld for approximately $20 \mathrm{hr}$ before threshold testing $5 \mathrm{~d}$ each week. All testing was done monocularly using controlled fixation, and neither monkey had more than $0.5 \mathrm{D}$ of refractive error in either eye.

\section{Placement of lesions}

After initial training on the psychophysical tasks, small ibotenic acid lesions were placed in magnocellular layer 1 of the left lateral geniculate nucleus of monkey 311 and the right geniculate of monkeys 602 and 856. For placement of the lesion, the monkey was sedated with ketamine $(15 \mathrm{mg} / \mathrm{kg}, \mathrm{i} . \mathrm{m}$.) and diazepam $(0.5 \mathrm{mg} / \mathrm{kg}, \mathrm{i} . \mathrm{m}$.). A catheter was then placed in a superficial leg vein, and the trachea was intubated. The animal was anesthetized with sufentanil citrate $(\sim 4 \mu \mathrm{g} / \mathrm{kg}$, i.v. $)$ and maintaincd on a sufentanil infusion for the remainder of the procedure (2-6 $\mu \mathrm{g} / \mathrm{kg} / \mathrm{hr}$ ). A small craniotomy and durotomy were made above the lateral geniculate nucleus using aseptic techniques. The animal was then paralyzed with Norcuron $(25 \mu \mathrm{g} / \mathrm{kg}$, i.v.; followed by an infusion at $100 \mu \mathrm{g} / \mathrm{kg} / \mathrm{hr}$ ) and respired at a rate and volume that maintained the partial pressure of expired $\mathrm{CO}_{2}$ between 25 and $40 \mathrm{~mm} \mathrm{Hg}$. Reflexes monitored during the $\sim 1 \mathrm{hr}$ surgery were used to determine the rate of sufentanil infusion during paralysis, and heart rate and expired $\mathrm{CO}_{2}$ were monitored throughout the procedure for signs of distress. A thermostatically controlled heating pad was used to warm the animal. The eyelids were held open with small retractors, and pupil reflexes and 
accommodation were blocked with Mydriacil. The corneas were covered with gas-permeable contact lenses, and the positions of the foveas were plotted using a reversing ophthalmoscope.

Recordings with platinum/iridium microelectrodes were used to locate the target visual field representation. The microelectrode was then replaced with a probe that could be used for both recording and injection. Physiological recordings of visual responses were again used to place the tip of the pipette in layer 1 (contralaterally driven magnocellular layer) of the lateral geniculate approximately $6^{\circ}$ eccentric along the horizontal meridian. Two microliters of ibotenic acid $(5 \mu \mathrm{g} / \mu \mathrm{l})$ were then pressure injected. The probe was then withdrawn and the paralytic infusion was stopped. The dura, bone, and skin were then closed using aseptic procedures. Recovery from paralysis was assisted with neostigmine $(0.24 \mathrm{mg} / \mathrm{kg}$, i.m.; preceded by atropine, $0.15 \mathrm{mg} / \mathrm{kg}, \mathrm{i} . \mathrm{m}$.).

\section{Implantation of scleral search coil and headmount}

After placement of the lesion, a scleral search coil was implanted in one eye of each monkey, under isoflurane anesthesia, so that eye position could be monitored, and a stainless-steel sleeve was attached to the skull so that the monkey's head could be immobilized (Judge et al., 1980). The coil was placed in the eye contralateral to the lesion, which was the eye tested psychophysically.

\section{Apparatus and procedures}

Contrast sensitivity. The seated monkey faced a 19-inch color monitor (Conrac 7211) at a distance of $211 \mathrm{~cm}$. The fixation spot was projected onto the face of the monitor, and contrast thresholds were measured with small patches of vertical grating displayed on the monitor. The grating targets were Gabor functions (cosinusoidal gratings multiplied by horizonal and vertical Gaussian weighting functions) generated on an Adage 3006 raster display unit and presented at a frame rate of 60 $\mathrm{Hz}$ (noninterlaced). The horizontal and vertical Gaussian weighting functions had equal space constants $\left(s=1.14^{\circ}\right)$. Thus, the grating was above $37 \%$ of peak contrast (full width at the $1 / e$ point) over a region of $2.28^{\circ}$.

All thresholds were tested monocularly using the eye contralateral to the lesion. This was necessary for assessing the magnocellular contribution selectively. Ibotenic acid lesions that destroyed an adequate volume of layer $\mathrm{l}$ invariably spread into other layers. The contralaterally driven parvocellular layer nearest the lesion is layer 4. Although small portions of layer 4 were always destroyed, careful physiological mapping showed that parvocellular damage was restricted to representations of the visual field away from the tested region (below). We did not test the ipsilateral eye hecause it is impossible to create an adequate magnocellular lesion (layer 2) without damaging the representation of the same part of the visual field in the neighboring parvocellular layer (layer 3).

While the monkey fixated within $\pm 0.3^{\circ}$ of a red fixation spot, the test stimulus was presented to the right or left of fixation (along the horizontal meridian of the retina), at a single location in each daily session. The interval between trials was 4 sec, correct choices (see below) were rewarded with fruit juice, incorrect choices were followed by a $6 \mathrm{sec}$ beeping tone, and fixation breaks or premature responses were followed by a $3 \mathrm{sec}$ beeping tone. The contrast (during contrast sensitivity testing) or the speed (during speed threshold testing) of the stimulus was varied according to a staircase, becoming easier by one step ( $2 \mathrm{~dB}$ contrast or 0.2 octave speed) after each error, and harder with probability 0.33 after each correct choice. Daily sessions consisted of 200 trials, and thresholds were taken at $75 \%$ correct responding either by linear interpolation or by probit fits to the daily psychometric functions (Finney, 1971).

The monitor displayed an equal-energy white background of $63 \mathrm{~cd} /$ $\mathrm{m}^{2}$, and Gabor functions were superimposed on this background for the following measures:

(1) Contrast sensitivity for the detection of drifting gratings [for $1 \mathrm{c} / \mathrm{deg}$ gratings drifting at 1,5 , or $20 \mathrm{deg} / \mathrm{sec}(1,5$, and $20 \mathrm{~Hz})]$. A yes-no procedure was used to test contrast thresholds. On each signal trial (presented randomly on half the trials), a vertical Gabor stimulus was presented during fixation, with contrast onset that followed a raised cosine of $0.5 \mathrm{~Hz}$, and remained on until either a fixation break or a response. The correct response was to the right push button on signal trials. On noise trials, no stimulus was presented, and the correct response was a press on the left push button. In the first experiment, contrast sensitivity was measured for stimuli drifting to the right (the horizontal Gaussian envelope remained fixed, and the cosinusoidal component moved to the right), and in the second experiment, for counterphase-modulated grating stimuli of the same spatial and temporal frequencies. Following these measures, sensitivity was tested at additional spatial and temporal frequencies in an attempt to determine if the reduced contrast sensitivity was due to the higher temporal frequency, the lower spatial frequency, or both. The additional points tested were $0.25 \mathrm{c} / \mathrm{deg}, 5 \mathrm{~Hz}$ and $4 \mathrm{c} / \mathrm{deg}, 20 \mathrm{~Hz}$.

(2) Contrast sensitivity for discriminating the direction of motion (of the same stimuli described above). On each trial, a Gabor stimulus was presented whose cosinusoidal component drifted either right or left, and the monkey was required to identify the dircction of motion by responding to the left or right push button. Stimulus contrast was varied from trial to trial as described above to determine a contrast threshold for this discrimination. Thresholds were tested for $1 \mathrm{c} / \mathrm{deg}$ spatial frequency and 1,5 , and $20 \mathrm{~Hz}$ temporal frequency.

Speed difference thresholds. A temporal forced choice task, like that described previously (Merigan and Maunsell, 1990), was used to determine difference thresholds for speed. Each trial began when the monkey fixated within $\pm 0.3^{\circ}$ of the fixation spot. Two tones, one of 2900 $\mathrm{Hz}$ and the other of $4500 \mathrm{~Hz}$, were presented successively to mark the two intervals of the temporal forced choice. A sinusoidal grating of 1 $\mathrm{c} /$ deg spatial frequency was presented on the display behind a $2.13^{\circ}$ diameter circular mask in a white surround that was illuminated at the mean luminance of the stimulus. The time course of the contrast of each stimulus followed a Gaussian envelope, centered on the middlc of each interval, with $s=0.3 \mathrm{sec}$. One of the two stimuli moved at the base speed for that condition, either 1,5 , or $20 \mathrm{deg} / \mathrm{sec}$. The comparison stimulus moved faster than the $1 \mathrm{or} 5 \mathrm{deg} / \mathrm{sec}$ base, but slower than the $20 \mathrm{deg} / \mathrm{sec}$ base speed. The monkey's task in all cases was to choose the faster-moving stimulus. The following controls were used to ensure that the discrimination was based on the speed of the two stimuli and not based on apparent contrast or extent of displacement. Use of apparent contrast was addressed by randomly varying the contrast of the stimuli to $+15 \%$ of the base contrast. Cues related to the extent or time of displacement were minimized by varying the time constant of the temporal Gaussian envelope by $\pm 29 \%$. Finally, we attempted to measure temporal frequency discrimination for monkey 602 in the lesion location using counterphase-modulated gratings of 0.8 contrast and $1 \mathrm{~cd} / \mathrm{deg}$ spatial frequency.

Monkey 311 was tested only at $1 \mathrm{deg} / \mathrm{sec}$. After a substantial elevation of speed threshold was found (see Fig. 3) at a contrast of 0.2 , contrasts of 0.4 were added to the test sequence. Monkey 602 showed no elevation of speed threshold at 1 and $5 \mathrm{deg} / \mathrm{sec}$ for a 0.2 contrast stimulus. After a large speed difference threshold elevation was found at $20 \mathrm{deg} / \mathrm{sec}$ for a 0.2 contrast stimulus, further thresholds were measured at 0.4 and 0.8 contrasts, with different contrasts interleaved to rule out gradual recovery. Since the higher contrasts eliminated the threshold elevation, control observations were then run for both this monkey and a human subject using counterphase-modulated gratings to test for the possibility that thresholds represented temporal frequency rather than speed discriminations.

\section{Reconstruction of lesions}

At the conclusion of behavioral testing, the lesion placed in monkey 602 was reconstructed with anatomical and physiological techniques. Immediately prior to death, a physiological map was made of the contralaterally driven layers $(1,4$, and 6$)$ in the right lateral geniculate nucleus. Electrolytic lesions $(10 \mu \mathrm{A} \times 10 \mathrm{sec})$ were made to facilitate reconstruction of the penetrations. After physiological recording, the monkey was killed and perfused with a saline rinse followed by $4 \%$ paraformaldehyde in phosphate-buffered saline. The brain was removed and blocked, and $40 \mu \mathrm{m}$ sections were cut on a freezing microtome. One section in four was reacted for cytochrome oxidase activity (WongRiley, 1979), and another stained with cresyl violet. The lesions were then reconstructed from these anatomical sections using the location of electrode penetrations to map them onto the visual field precisely. The lesion in the second animal (monkey 311 ) was described previously (Merigan and Maunsell, 1990). Monkey 856 is still being tested; the lesion will be reconstructed when testing is complete.

\section{Results}

The location and extent of the lateral geniculate lesion in monkey 602 are illustrated in Figure 1. Each panel shows the central portion of the contralateral visual field representation in a dif- 

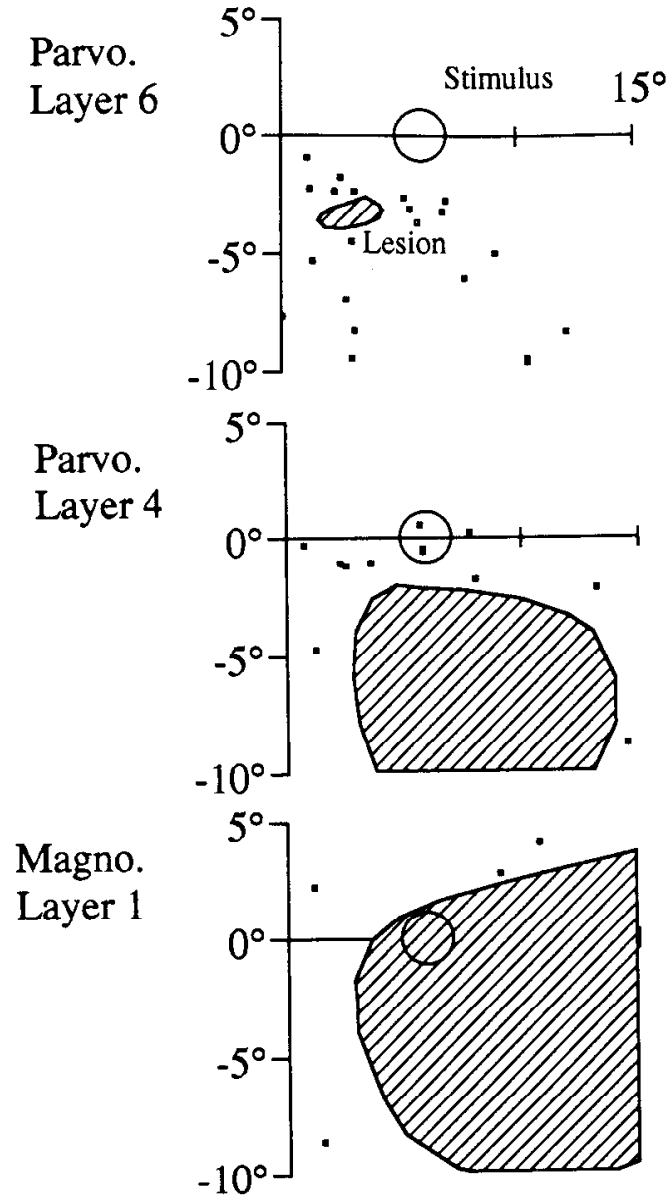

Figure 1. Visual field maps showing the portion of the visual field representation lesioned in different geniculate layers in monkey 602 . The horizontal and vertical lines represent the horizontal and vertical meridia of the visual field, and the fixation point is at their intersection. A circle on each plot shows the location and extent of the test stimulus, squares show physiological recording sites where normal activity was found, and the hatched area shows a reconstruction of the lesion from anatomical sections and physiological mapping. The number of receptive fields plotted was 27 in layer 6,15 in layer 4 , and 18 in layer 1 .

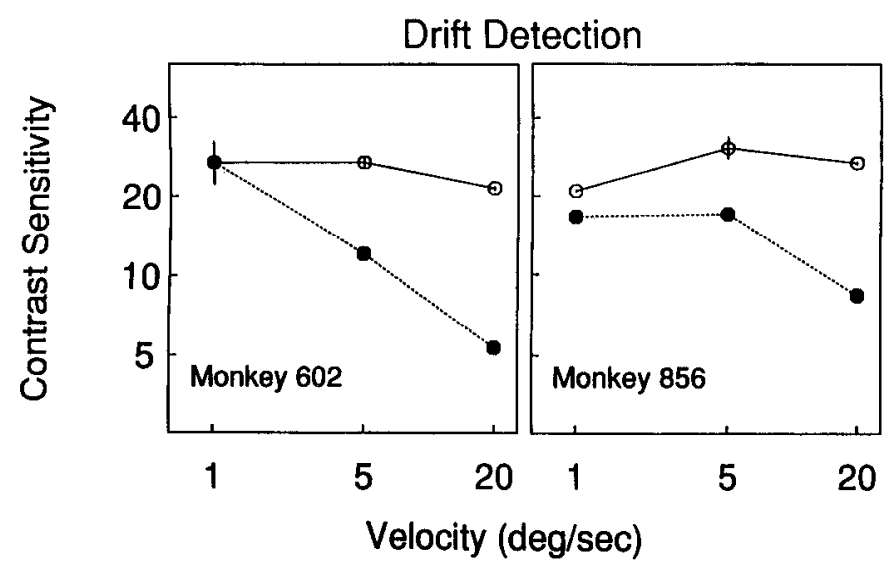

Figure 2. Effect of M pathway lesions on contrast sensitivity for detecting drifting Gabor patches moving at different velocities. Open symbols show results measured in an intact portion of the visual field, and solid symbols, for a portion of the field where the M pathway was interrupted by an ibotenic acid lesion.

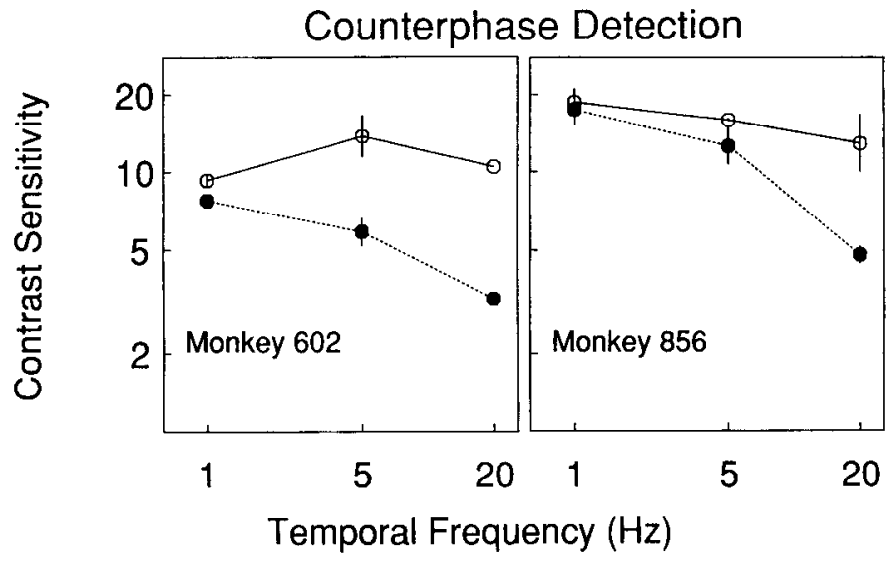

Figure 3. Same as Figure 2 except that this figure shows sensitivity for detecting counterphase-modulated gratings.

ferent geniculate layer. Some of the receptive field centers that were plotted during the final mapping session are marked with squares. The borders of the lesion were located in a series of histological sections and related to visual field locus by interpolating between nearby recording sites. Hatched regions with a border indicate representations completely destroyed by the lesion. The circle on each horizontal meridian shows the location and size of the test stimulus for contrast sensitivity. The ibotenic acid spread into noninjected layers and was particularly prone to spread up the electrode track. However, unintended damage to other contralaterally driven layers was restricted to untested parts of the visual field, and the lesion completely destroyed the stimulus representation in target layers while completely sparing the nontarget layers. The lesion in monkey 311 (Merigan and Maunsell, 1990) also completely destroyed magnocellular layer 1 in the tested portion of the visual field and completely spared the corresponding representation in both layers 4 and 6 of parvocellular geniculate.

Figure 2 shows contrast sensitivity for detccting the presence of drifting patches of grating. The Gaussian weighting function remained fixed in place, and the cosinusoidal component of the Gabor drifted to the right. Data for the intact visual field (opposite the lesioned side) are shown as open symbols, and for the magnocellular-lesioned portion by solid symbols. The magnocellular lesion caused little or no deficit in sensitivity at the lowesl velocily, but the loss was substantial at higher velocities, reaching 0.52 and $0.60 \log$ units at $20 \mathrm{deg} / \mathrm{sec}$ for monkeys 856 and 602 , respectively.

The detection contrast sensitivities shown in Figure 3 were measured with stimuli of the same spatial and temporal frequencies as in Figure 2, but they were sinusoidally counterphase modulated rather than drifting. Solid symbols show data from visual field locations corresponding to magnocellular lesions, and open symbols, from the opposite visual hemifield. Overall sensitivities were lower than for drifting stimuli, but again, $M$ pathway lesions caused decreases in sensitivity only at the higher temporal frequencies.

Log loss of contrast sensitivity for detecting patches of drifting grating is shown in Figure 4. The spatial frequency axis in this figure extends to about $30 \mathrm{c} / \mathrm{deg}$, which is probably beyond the spatial resolution of the monkey at this eccentricity. We expect that the monkey's acuity [and thus, the limit of $\mathrm{P}$ (parvocellular) pathway response] would be about $20 \mathrm{c} / \mathrm{deg}$ (Merigan 

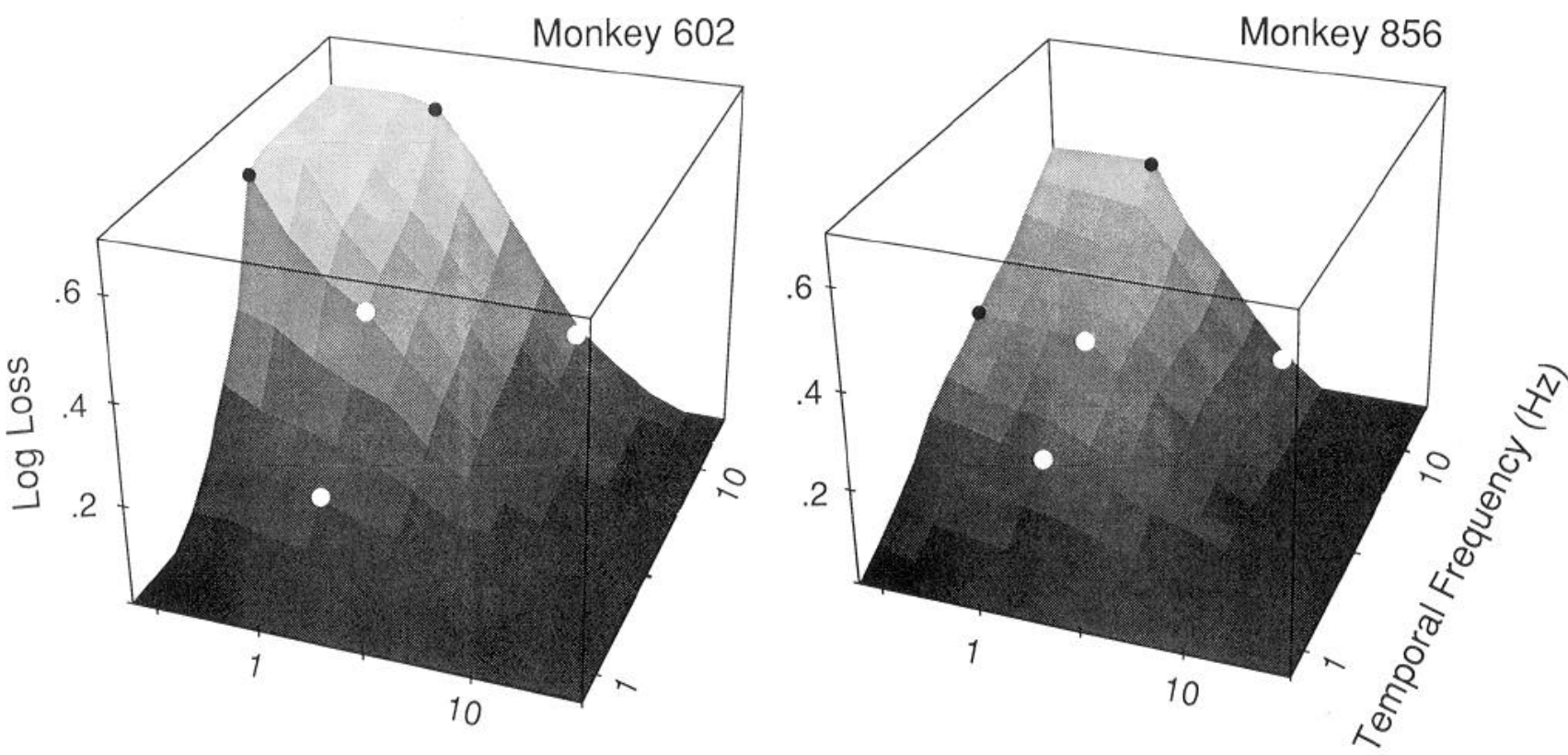

Spatial Frequency (c/deg)

Figure 4. Loss of sensitivity for detecting drifting gratings produced by magnocellular lesions. Measured values are shown by solid circles; the surface was interpolated from these data points.

et al., 1991a) under the conditions used here, and that this resolution would be rather independent of temporal frequency (Kelly, 1979). This figure includes the data shown in Figure 2 as well as additional conditions listed in Materials and Methods. Sensitivity loss was measured for combinations of spatial and temporal frequencies indicated by the five small circles, and the surface was fit by interpolation and extrapolation from these points. The effect of magnocellular lesions in both monkeys was to decrease sensitivity to stimuli of lower spatial and higher temporal frequency.

Figure 5 shows the contrast sensitivity of monkeys 602 and 856 for discriminating the direction of motion of drifting patches of grating. The Gaussian weighting functions remained fixed, and the cosinusoidal component of the Gabor function drifted either to the right or left on each trial. Stimuli of three different velocities were tested, and results are shown for the intact (open symbols) and M pathway-lesioned (solid symbols) portions of the visual field. At higher speeds, both monkeys showed a progressive loss in sensitivity in the lesioned part of the visual field. Loss for gratings drifting at $20 \mathrm{deg} / \mathrm{sec}$ was 0.44 and $0.62 \mathrm{log}$ units for monkeys 856 and 602 , respectively. The magnitude and the speed dependence of the loss shown in this figure are similar to those shown for detection of drifting and counterphase-modulated gratings in Figures 2 and 3.

The effect of $\mathrm{M}$ pathway lesions on speed difference thresholds is shown in Figure 6. Discrimination sensitivity for the speed of grating motion is shown as the Weber fraction (speed difference/base speed). The parameter is peak contrast of the Gabor functions; $0.2=$ squares, $0.4=$ circles, and $0.8=$ triangles. Lesions of the M pathway (solid symbols) greatly elevated speed difference thresholds for stimulus contrasts of 0.2 for monkey 311 at $1 \mathrm{deg} / \mathrm{sec}$ and for monkey 602 at $20 \mathrm{deg} / \mathrm{sec}$. However, when stimulus contrast was raised under these conditions, the elevation of speed difference thresholds decreased or disappeared entirely.
Control measures of temporal frequency discrimination were made in monkey 602 in the magnocellular-lesioned portion of the visual field using 0.8 contrast counterphase-modulated gratings of $1 \mathrm{c} / \mathrm{deg}$ and of $20 \mathrm{~Hz}$ base temporal frequency. No threshold could be obtained after three sessions of testing with temporal frequency ratios up to $1: 3$.

\section{Discussion}

This study demonstrates that the visibility of stimuli of low spatial and high temporal frequencies depends strongly on the $M$ pathway. When the stimuli are drifting gratings, high temporal and low spatial frequencies produce high speeds, and as shown in Figure 2, the visual contribution of the M pathway increases as speed is raised. On the other hand, this specialization of the M pathway is not specific to moving stimuli, since

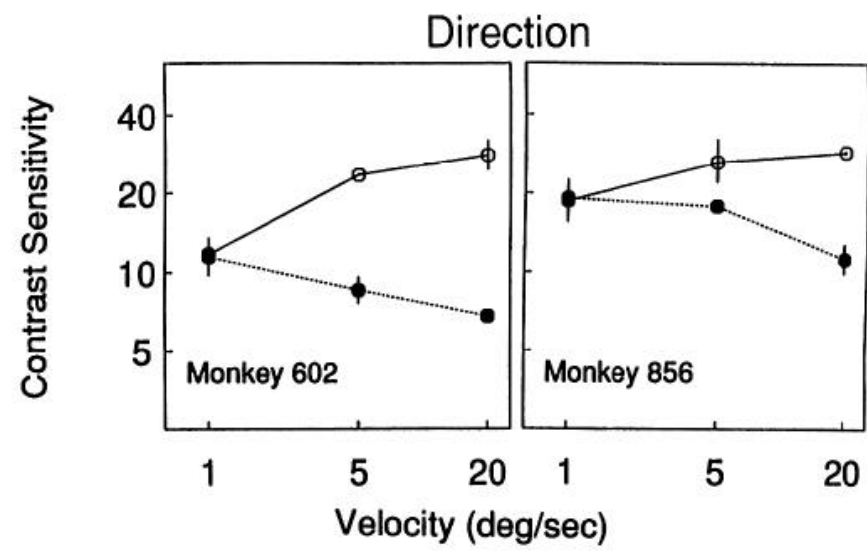

Figure 5. Effect of $\mathrm{M}$ pathway lesions on contrast sensitivity for discriminating the direction of motion of drifting Gabor patches moving at different velocities. For other conventions, see Figure 2. 


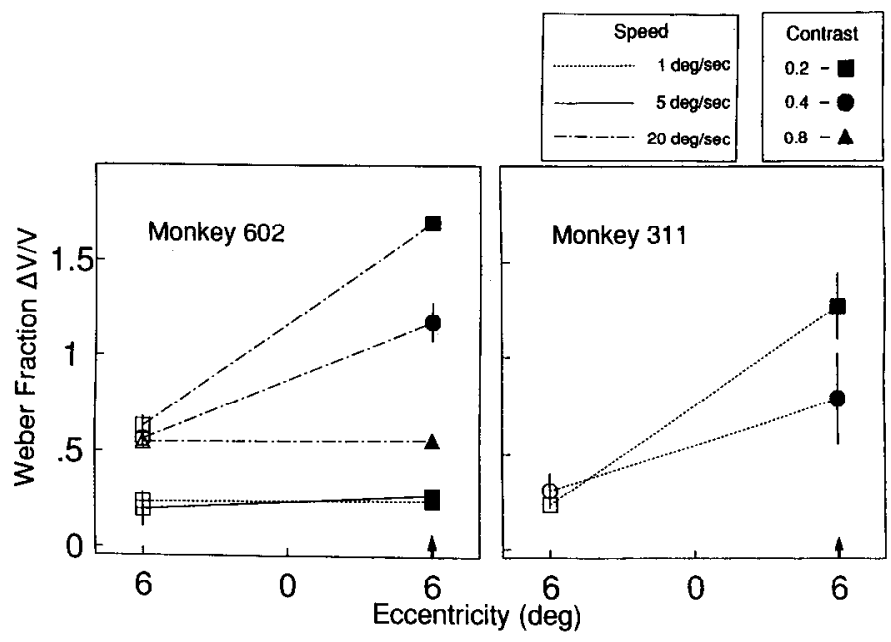

Figure 6. Weber fractions $(\Delta \mathrm{v} / \mathrm{v})$ for speed discrimination for two monkeys in control (open symbols) and $\mathrm{M}$ pathway-lesioned (solid sym$b o l s$ ) portions of the visual field. Control measures were taken at $6^{\circ}$ in the nasal visual field, and the magnocellular lesion measures, at $6^{\circ}$ in the temporal visual field. Speed difference thresholds were measured for only a single base speed for monkey $311(1 \mathrm{deg} / \mathrm{sec} ;$ dotted lines $)$ but at three base speeds for monkey 602 [1 (dotted), 5 (solid), and 20 $\mathrm{deg} / \mathrm{sec}$ (dot-dash lines)]. Initial measurements were made at a contrast of 0.2 (squares), but then interleaved measurements were made at contrasts of 0.4 (circles) and 0.8 (triangles) under conditions where Weber fractions were elevated. Comparison stimuli moved faster than the 1 and $5 \mathrm{deg} / \mathrm{sec}$ base speeds, but slower than the $20 \mathrm{deg} / \mathrm{sec}$ speed. Error bars are $\pm \mathrm{SD}$.

we found exactly the same dependence on low spatial and high temporal frequencies for the detection of counterphase-modulated gratings. Sensitivity for opposite direction discrimination was also reduced at high speeds after lesions of the $M$ pathway. However, this result does not suggest a motion-specific contribution of the M pathway, since the reduced discrimination sensitivity paralleled that found for detection sensitivity, and the magnocellular-lesioned monkeys could make direction discriminations near contrast threshold. We also found that speed difference thresholds were elevated under low-contrast conditions by $M$ pathway lesions, a result that could simply reflect the reduced visibility of the test stimuli. When the contrast of the gratings used to test speed difference thresholds was raised, the discrimination deficit disappeared. Together, these findings suggest that the $\mathrm{M}$ pathway is important primarily for mediating the visibility of low spatial and high temporal frequencies. This pathway is not necessary for the discrimination of the most important features of motion perception, direction and speed. Thus, the important contribution of the $\mathrm{M}$ pathway to motion processing may simply reflect the utility for motion perception of the spatiotemporal frequencies it transmits.

\section{Spatiotemporal contrast sensitivity}

Lesion studies do not provide direct measurements of the sensitivity of individual pathways. Rather, tests of contrast sensitivity after magnocellular lesions only show the sensitivity of the remaining major pathway, the $P$ pathway [we believe that only the $\mathrm{M}$ and $\mathrm{P}$ pathways contribute to contrast thresholds (Schiller et al., 1990)]. Thus, results shown here after M pathway lesions (Figs. 2, 3, 5) probably reflect the sensitivity of the $P$ pathway, and indicate that this sensitivity declines greatly at low spatial and high temporal frequencies. Under those conditions where sensitivity was reduced following an $\mathbf{M}$ pathway lesion, we can probably attribute the prelesion sensitivity to the $M$ pathway. In addition, residual sensitivity after $P$ pathway lesions in previous studies (Merigan and Eskin, 1986; Merigan et al., 1991a) is also likely due to the M pathway. Together, the present study and these previous results indicate that the $M$ pathway has its greatest sensitivity at low spatial and high temporal frequencies, while the $P$ pathway has very poor sensitivity for these frequencies (Merigan, 1991).

We determined in an earlier study (Merigan et al., 1991a) that chromatic information appears to be carried only by the $P$ pathway, and this appears to be true even at the low spatial frequencies where the $\mathrm{M}$ pathway dominates luminance vision. Beyond this specialization for chromatic stimulation, the little available evidence suggests that the $P$ and $M$ pathways are not specialized for particular visual capacities such as stereo or contrast perception (Merigan, 1991). Rather, the two pathways seem to differ primarily in the spatiotemporal information they convey. This specialization of subcortical visual pathways for different regions of spatiotemporal frequency space is consistent with several well-known anatomical and physiological features of these pathways. Anatomical characteristics that agree with these results are the much greater number, and hence the finer sampling density, of $\mathbf{P}$ than $\mathbf{M}$ ganglion cells and the approximately eightfold larger dendritic fields of $M$ than $P$ ganglion cells (Perry et al., 1984). Physiological findings that parallel these results are the slightly higher temporal frequency response of $\mathrm{M}$ than $\mathbf{P}$ ganglion cells (Gouras and Zrenner, 1979) and lateral geniculate neurons (Hicks et al., 1983; Derrington and Lennie, 1984), and the relatively poor low temporal frequency response of lateral geniculate magnocellular neurons (Purpura et al., 1990).

The present identification of $P$ and $M$ pathways with different spatiotemporal frequencies invites comparisons with the longstanding distinction, based largely on psychophysical measurements in humans and monkeys, between "transient" and "sustained" visual mechanisms (Keesey, 1972; Kulikowski and Tolhurst, 1973; Harwerth et al., 1980). These reports have attributed several visual phenomena to the parallel activity of two postulated visual channels: a "sustained" channel that is most responsive at lower temporal and higher spatial frequencies, and a "transient" channel that is most responsive at higher temporal and lower spatial frequencies. According to this view, when perception at contrast threshold is mediated by the "transient" channel, motion or flicker of the stimulus is visible, but not its spatial structure, and subthreshold masking is most effective at the onset and offset of the masking stimulus. On the other hand, when perception at contrast threshold is mediated by the "sustained" channel, the spatial pattern of the stimulus is visible, but not its flicker or motion, and subthreshold masking is equally effective throughout the duration of the masking stimulus. Additional evidence for such a two-mechanism model is that the functions relating contrast thresholds to stimulus duration, and reaction time to stimulus contrast, have two branches, and the spatiotemporal locations of these branches suggest that they correspond to the two mechanisms. It is quite clear from a comparison of the above reports with the present that the sensitivity of the $M$ pathway is like that described for the "transient" channel and that the sensitivity of the $P$ pathway is similar to that of the "sustained" channel (Anderson and Burr, 1985). This can be seen directly by comparing Figure 2 in Harris (1980) with Figure 4 of the present article. Other features of these channels also suggest the characteristics of the $M$ and $P$ pathways, for example, the masking at onset and offset that is a characteristic of the "transient" channel may parallel the higher 
temporal frequency response of the $\mathbf{M}$ pathway. Unfortunately, as the present study makes clear, specially designed studies are needed to disentangle the contributions of a subcortical pathway from those of the cortical areas to which it projects. No studies have yet been carried out that examine whether the $\mathbf{P}$ and $\mathbf{M}$ pathways might be the substrate of "sustained" and "transient" mechanisms, or whether these "channels" simply reflect the response of cortical areas to stimuli moving at different speeds.

\section{Direction discrimination}

There is now substantial evidence that, at contrast threshold, visual sensitivity is mediated by motion-sensitive mechanisms at low spatial and high temporal frequencies, but by nonmotion mechanisms at higher spatial and lower temporal frequencies (Pasternak and Leinen, 1986; Pasternak, 1987; Watson et al., 1980). This is important to the present study because the conditions under which detection has been reported to be mediated by motion-sensitive mechanisms are the same conditions under which threshold detection in the present study was dominated by the M pathway. Two types of evidence have indicated detection by motion-sensitive mechanisms. The first is the comparison of contrast thresholds for the detection of moving gratings and for discrimination of opposite directions of motion. Watson and Robson (1981) have proposed that if some stimulus property, such as spatial or temporal frequency, can be discriminated at detection threshold, then the detectors mediating threshold must be "labeled" for that stimulus property. In fact, it appears that opposite directions of motion can be discriminated at contrast threshold for the detection of moving gratings (Watson et al., 1980; Green, 1983), but only for low spatial and high temporal frequencies. [While this is true for opposite directions, suprathreshold contrast is required for direction discriminations that involve narrower ranges of directions (Ball et al., 1983).] On the other hand, at lower temporal and higher spatial frequencies, stimuli must be above detection threshold before direction can be discriminated. These results indicate two visual detection mechanisms with overlapping spatiotemporal sensitivities, but with greater sensitivity of the motion mechanism at higher speeds, and higher sensitivity of the nonmotion mechanism at lower speeds. This pattern can be seen clearly in the data of monkey 602 by comparing the control data from Figures 2 and 5. The monkey can discriminate direction at $\mathrm{I} \mathrm{deg} / \mathrm{sec}$ only at contrasts more than twofold higher than that needed to detect the grating. However, at 5 and 20 $\mathrm{deg} / \mathrm{sec}$, detection and direction thresholds are similar. Monkey 856 shows little direction-detection difference even at $1 \mathrm{deg} /$ sec, although such a difference would likely be present at yet lower velocities (Watson et al., 1980).

A second type of evidence for motion-sensitive mechanisms comes from contrast summation experiments (Levinson and Sekuler, 1975; Watson et al., 1980; Stromeyer et al., 1984). These studies have shown that, for stimuli of higher temporal and lower spatial frequencies, there is little or no summation between stimuli moving in opposite directions, that is, that the detecting mechanism was directional. On the other hand, at lower temporal and higher spatial frequencies, strong summation was observed between opposite direction stimuli, indicating detection by nondirectional mechanisms.

In addition to the observation that the spatiotemporal tuning of the M pathway parallels that of direction-sensitive motion mechanisms, there are other bases for suspecting a relationship of the M pathway to motion mechanisms. First, direction sensitivity in area V1 of the monkey is very pronounced in layers
$4 \mathrm{C} \alpha$ and $4 \mathrm{~B}$ that receive input from the magnocellular lateral geniculate, but very weak in layer $4 \mathrm{C} \beta$ that receives input from parvocellular lateral geniculate (Hawken and Parker, 1984). This difference assumes some importance from the finding that the virtual abolition of cortical directional selectivity by visual deprivation during rearing (Pasternak and Leinen, 1986) devastates opposite direction discrimination at low contrasts. Second, the cortical areas that receive input from magnocellular geniculate, $4 \mathrm{C} \alpha$ and 4B, provide the major input to cortical areas MT and MST (Maunsell and Newsome, 1987), which are thought to be important for motion processing (Movshon et al., 1985; Newsome and Paré, 1988; Merigan et al., 1991b; Pasternak et al., 1991). Finally, it has been shown that lesions of the $M$ pathway abolished the detection of motion of a group of dots that were embedded in a field of random dots (Schiller et al., 1990).

Despite all this evidence that motion perception could depend on the M pathway, the present study shows clearly that direction discrimination survives lesions of the $\mathbf{M}$ pathway. Furthermore, direction could be discriminated at contrast threshold after $\mathrm{M}$ pathway lesions, despite a large increase in contrast threshold. This indicates that the P pathway functions as a "labeled" detector for direction of motion and that the increase in contrast threshold for direction was due simply to the poorer sensitivity of the $P$ than $M$ pathway for particular spatiotemporal frequencies. It appears that the greater sensitivity of motion mechanisms at higher stimulus speeds may stem from the properties of cortical motion-sensing mechanisms that receive input from both $\mathrm{M}$ and $\mathrm{P}$ subcortical pathways (Maunsell et al., 1990). The close correspondence between the spatiotemporal sensitivity of directional mechanisms and that of the $M$ pathway, as well as the large projection of the M pathway to cortical areas MT and MST, may simply reflect the great utility of certain spatiotemporal frequencies for motion processing. Finally, the finding of Schiller et al. (1990) that motion detection was abolished by $\mathrm{M}$ pathway lesions does not necessarily reflect a loss of motion perception. The present results suggest that the Schiller finding may represent severely reduced visibility of the moving dot pattern, sufficient to have eliminated the detection of motion.

\section{Speed discrimination}

The second major parameter of motion perception, in addition to direction, is the speed of visual motion. Recent psychophysical experiments have established that speed of motion can be directly perceived, that is, that it does not have to be calculated from temporal frequency, extent of displacement, etc. (McKee et al., 1986; Pasternak, 1987). Inlike direction discrimination, speed discrimination does not simply improve toward lower spatial and higher temporal frequencies (as does the sensitivity of the $M$ pathway) but rather has an optimal spatiotemporal frequency at each velocity (McKee et al., 1986; DeBruyn and Orban, 1988; Panish, 1988). However, at higher spatial and lower temporal frequencies (where the sensitivity of the $M$ pathway is very poor), speed discrimination appears to be limited to those conditions for which direction discrimination is possible (Pasternak, 1987).

The speed discrimination thresholds shown in Figure 6 for the nonlesion portion of the visual field range from Weber fractions of about 0.2 for $1 \mathrm{deg} / \mathrm{sec}$ base speed to about 0.5 for 20 $\mathrm{deg} / \mathrm{sec}$ base speed. These thresholds are somewhat higher than the values of $0.05-0.1$ reported for human (McKee et al., 1986; Pasternak, 1987; DeBruyn and Orban, 1988; Panish, 1988) or 0.1 for monkey (Merigan et al., 1991b) foveal thresholds. The 
major factors in this difference appear to be the eccentric location $\left(6^{\circ}\right)$ and brief duration (Gaussian with $s=0.3 \mathrm{sec}$ ) of the test grating. However, despite the higher thresholds measured under these conditions, it is quite clear, given the precautions outlined in Materials and Methods, that these thresholds reflect speed discrimination and that they were not influenced by apparent contrast or displacement of stimuli. We also tested temporal frequency discrimination thresholds in monkey 602 (Results) with counterphase-modulated gratings at $20 \mathrm{~Hz}$ and were unable to obtain thresholds with temporal frequency ratios up to $1: 3$, suggesting that the dramatic improvement in Weber fractions for this monkey at 0.8 contrast was not due to the discrimination of temporal frequency or flicker at the leading or trailing edge of the drifting grating.

Speed discrimination thresholds were greatly elevated for monkey 311 at $1 \mathrm{deg} / \mathrm{sec}$ and for monkey 602 at $20 \mathrm{deg} / \mathrm{sec}$. The latter effect was clearly consistent with the contrast thresholds measured in this study. At $1 \mathrm{deg} / \mathrm{sec}$, the detection threshold for monkey 602 was not affected by the lesion, and at $5 \mathrm{deg} /$ sec, it was elevated by about a factor of 2 , but under neither condition were speed difference thresholds elevated. At a speed of $20 \mathrm{deg} / \mathrm{sec}$, monkey 602's contrast threshold was raised about fourfold, following the lesion, to a contrast of about 0.2 , the same contrast as that of the grating stimulus used to test speed threshold. This resulted in a threefold elevation of the speed threshold, which then recovered to control values when the stimulus contrast was raised to 0.8 . Monkey 311 showed a large elevation of speed threshold at $1 \mathrm{deg} / \mathrm{sec}$, which was also partially ameliorated by raising stimulus contrast to 0.4 . For this stimulus condition, monkey 602 showed neither an increase in contrast threshold nor an increase in speed threshold, and monkey 856 showed little or no effect on contrast threshold. Unfortunately, we did not measure monkey 311 's contrast sensitivity under these stimulus conditions, but she showed lower sensitivity for higher temporal frequency drifting gratings than monkey 602 (see Merigan and Maunsell, 1990), and it appears likely that the effect of the magnocellular lesion was to reduce her contrast sensitivity at $1 \mathrm{deg} / \mathrm{sec}$, especially since doubling the contrast of the drifting grating greatly improved her Weber fraction for speed.

Like our earlier results for direction discrimination, our speed discrimination testing shows that the monkey can discriminate speed of stimulus motion entirely on the basis of input from the P pathway. Some elevations of speed threshold resulted from $M$ pathway lesions, but they appeared to be due entirely to the decreased visibility of the moving stimuli after $M$ pathway lesions. These experiments demonstrate that the two primary features of motion perception, direction and speed, can be discriminated using only information conveyed by the $P$ pathway (i.e., after $M$ pathway lesions in this study), although in the absence of lesions, this discrimination is probably made using information conveyed by the $\mathrm{M}$ pathway, which is more sensitive at higher speeds of motion. As is true for other aspects of visual processing (Merigan, 1991), the $P$ and $M$ pathways appear not to be exclusive conduits for visual motion information.

\section{References}

Anderson SJ, Burr DC (1985) Spatial and temporal selectivity of the human motion detection system. Vision Res 25:1147-1154.

Ball K, Sekuler R, Machamer J (1983) Detection and identification of moving targets. Vision Res 23:229-238.
DeBruyn B, Orban G (1988) Human velocity and direction discrimination measured with random dot patterns. Vision Res 12:13231335.

Derrington AM, Lennie P (1984) Spatial and temporal contrast sensitivities of neurons in lateral geniculate nucleus of macaque. J Physiol (Lond) 357:219-240.

DcYoc EA, Van Essen DC (1988) Concurrent processing streams in monkey visual cortex. Trends Neurosci 11:219-226.

Finney DJ (1971) Probit analysis. Cambridge: Cambridge UP.

Gouras P, Zrenner E (1979) Enhancement of liminance flicker by color-opponent mechanisms. Science 205:587-589.

Green M (1983) Contrast detection and direction discrimination of drifting gratings. Vision Res 23:281-289.

Harris MG (1980) Velocity specificity of the flicker to pattern sensitivity ratio in human vision. Vision Res 20:687-691.

Harwerth RS, Boltz RL, Smith EL (1980) Psychophysical evidence for sustained and transient channels in the monkey visual system. Vision Res 20:15-22.

Hawken MJ, Parker AJ (1984) Contrast sensitivity and orientation selectivity in lamina $1 \mathrm{~V}$ of the striate cortex of old world monkeys. Exp Brain Res 54:367-372.

Hicks TP, Lee BB, Vidyasagar TR (1983) The responses of cells in the macaque lateral geniculate nucleus to sinusoidal gratings. J Physiol (Lond) 337:183-200.

Judge SJ, Richmond BJ, Chu FC (1980) Implantation of magnetic search coils for measurement of eye position: an improved method. Vision Res 20:535-538.

Keesey UT (1972) Flicker and pattern detection: a comparison of thresholds. J Opt Soc Am 62:446-448.

Kelly DH (1979) Motion and vision. II. Stabilized spatiotemporal threshold surface. J Opt Soc Am 69:1340-1349.

Kulikowski JJ, Tolhurst DJ (1973) Psychophysical evidence for sustained and transient detectors in human vision. J Physiol (Lond) 232: 149-162.

Levinson E, Sekuler $\mathbf{R}$ (1975) The independence of channels in human vision selective for direction of movement. J Physiol (Lond) 250: 347-366.

Maunsell JHR, Newsome WT (1987) Visual processing in monkey extrastriate cortex. Annu Rev Neurosci 10:363-401.

Maunsell JHR, Nealey TA, DePriest DD (1990) Magnocellular and parvocellular contributions to responses in the middle temporal visual area (MT) of the macaque monkey. J Neurosci 10:3323-3334.

McKee SP, Silverman GH, Nakayama K (1986) Precise velocity discrimination despite random variation in temporal frequency and contrast. Vision Res 26:609-619.

Merigan WH (1991) P and M pathway specialization in the macaque. In: From pigments to perception: advances in understanding visual processes (Valberg A, and Lee BB, eds), New York: Plenum.

Merigan WH, Eskin TA (1986) Spatio-temporal vision of macaques with severe loss of $\mathrm{Pb}$ retinal ganglion cells. Vision Res 26:17511761.

Merigan WH, Maunsell JHR (1990) Macaque vision after magnocellular lateral geniculate lesions. Visual Neurosci 5:347-352.

Merigan WH, Katz LM, Maunsell JHR (1991a) The effects of parvocellular lateral geniculate lesions on the acuity and contrast sensitivity of macaque monkeys. J Neurosci 11:994-1001.

Merigan WH, Pasternak T, Polashenski W, Maunsell JHR (1991b) Permanent deficits in speed discrimination after MT/MST lesions in a macaque monkey. Invest Ophthalmol Vis Sci [Suppl] 32:824.

Movshon JA, Adelson EH, Gizzi MS, Newsome W (1985) The analysis of moving patterns. Exp Brain Res [Suppl] 11:117-151.

Newsome WT, Paré EB (1988) A selective impairment of motion perception following lesions of the middle temporal area (MT). J Neurosci 8:2201-2211.

Panish SC (1988) Velocity discrimination at constant multiples of threshold contrast. Vision Res 28:193-201.

Pasternak T (1987) Discrimination of differences in speed and flicker rate depends on directionally selective mechanisms. Vision Res 27: 1881-1890.

Pasternak T, Leinen L (1986) Pattern and motion vision in cats with selective loss of cortical directional sclcctivity. J Ncurosci 6:938-945.

Pasternak T, Maunsell JHR, Polashenski W, Merigan WH (1991) Deficits in global motion perception after MT/MST lesions in a macaque. Invest Ophthalmol Vis Sci [Suppl] 32:824. 
Perry VH, Oehler R, Cowey A (1984) Retinal ganglion cells which project to the dorsal lateral geniculate nucleus in the macaque monkey. Neuroscience 12:1101-1123.

Purpura K, Tranchina D, Kaplan E, Shapley RM (1990) Light adaptation in the primate retina: analysis of changes in gain and dynamics of monkey retinal ganglion cells. Visual Neurosci 4:75-93.

Schiller PH, Logothetis NK, Charles ER (1990) Functions of the colour-opponent and broad-band channels of the visual system. Nature 343:68-70.

Stromeyer CF, Kronauer RE, Madsen JC, Klein SA (1984) Opponentmovement mechanisms in human vision. J Opt Soc Am 1:876-884.
Watson AB, Robson JG (1981) Discrimination at threshold: labelled detectors in human vision. Vision Res 21:1115-1122.

Watson AB, Thompson PG, Murphy BJ, Nachmias J (1980) Summation and discrimination of gratings moving in opposite directions. Vision Res 20:341-347.

Wong-Riley $M$ (1979) Changes in the visual system of monocularly sutured or enucleated cats demonstrable with cytochrome-oxidase histochemistry. Brain Res 171:11-28. 